\title{
Robust CERN LHC Higgs boson search in weak boson fusion
}

\author{
Alexandre Alves* \\ Instituto de Física Teórica, UNESP, São Paulo, Brazil \\ Oscar Éboli ${ }^{\dagger}$ \\ Instituto de Física, Universidade de São Paulo, São Paulo, SP, Brazil \\ Tilman Plehn \\ Theory Division, CERN, CH-1211 Geneva 23, Switzerland \\ David Rainwater ${ }^{\S}$ \\ DESY-T-, Notkestrasse 85, D-22603 Hamburg, Germany \\ (Received 4 September 2003; published 12 April 2004)
}

\begin{abstract}
We demonstrate that a CERN LHC Higgs boson search in weak boson fusion production with subsequent decay to weak boson pairs is robust against extensions of the standard model or minimal supersymmetric standard model involving a large number of Higgs doublets. We also show that the transverse mass distribution provides unambiguous discrimination of a continuum Higgs signal from the standard model.
\end{abstract}

DOI: 10.1103/PhysRevD.69.075005

PACS number(s): 14.80.Cp, 14.80.Bn

\section{INTRODUCTION}

The search for the origin of electroweak symmetry breaking and fermion mass generation, generally believed to be one or more scalar $S U(2)_{L}$ Higgs doublets, remains one of the premier tasks of high energy physics. Fits to precision electroweak data have for some time suggested a relatively small Higgs boson mass, in case of the standard model (SM) of order $m_{h} \lesssim 200 \mathrm{GeV}$ [1].

The CERN Large Hadron Collider (LHC) will have the capability to search for physical Higgs boson(s) over a huge mass range. In the SM case it will have multiple coverage of search channels for any given Higgs boson mass, in particular for the intermediate mass range [2-9]. This coverage relies heavily on Higgs production via weak boson fusion (WBF). The advantage of WBF, where the scattered finalstate quarks receive significant transverse momentum and are observed in the detector as far-forward/backward jets, is the strong reduction of QCD backgrounds due to the kinematical configuration of the colored part of the event. In the minimal supersymmetric standard model (MSSM), all five of the physical Higgs bosons may not be observable over all of MSSM parameter space. However, a well-established nolose theorem guarantees that at least one of the two $C P$-even neutral states will be seen in WBF with subsequent decay to either tau leptons or gauge bosons, after taking into account the CERN $e^{+} e^{-}$collider LEP limits [10]. For even more complex models there exists a No-Lose theorem for the NMSSM (NMSSM) [11], which also relies on WBF Higgs production, but includes decay modes which do not occur in simpler models.

\footnotetext{
*Email address: aalves@ift.unesp.br

†Email address: eboli@fma.if.usp.br

\#Email address: tilman.plehn@cern.ch

${ }^{\S}$ Email address: david.rainwater@desy.de
}

However, the set of possible symmetry breaking scenarios is much larger than these models, and it is natural to consider whether the LHC can similarly guarantee discovery for other cases. A popular way to try to hide a Higgs sector from collider searches is via additional fields. These can have two effects: first, one can try to reduce the branching fractions to observable decay modes, in analogy to invisible Higgs decays, for example in supersymmetry. The so-called stealth model [12] achieves this through the presence of additional singlets, which couple (strongly) to the Higgs boson and lead to a large invisible decay width. The search for these particles is tedious for two reasons, the invisible decay and their huge width, which vastly exceeds the experimental mass resolution and hence becomes a limiting factor in background suppression. Nevertheless, the invisible decay should in principle be observable at the LHC in weak boson fusion, because the recoiling tagging jets are not sensitive to the Higgs boson mass, as long as the particle is in the intermediate mass range [13].

Another alternative scenario inflates the Higgs signal width via a large number of Higgs doublets, with the measured vacuum expectation value distributed among them [14]. Multiple $C P$-even neutral Higgs bosons exist, each with diminished coupling to weak bosons. The WBF production rate of any one of these can be suppressed considerably, such that by itself it would be lost in the background. To make matters worse, each Higgs boson in general has a different mass, forming a broad spectrum of states. Poor LHC detector resolution may smear the individual peaks out to a smooth continuum. These models lead to the speculation that the Higgs sector may be unidentifiable at the LHC, even though high-energy $W W$ scattering would clearly observe unitarity [15].

To decide whether there really is a hole in the LHC Higgs boson discovery potential, we investigate these continuum models in detail. Our analysis of WBF production of such a continuum of Higgs bosons, followed by decay to dileptons 
$\left(e^{ \pm} \mu^{\mp}, e^{ \pm} e^{\mp}, \mu^{ \pm} \mu^{\mp}\right)$ via a pair of $W$ bosons confirms the robustness of the WBF Higgs searches in the case of nonstandard Higgs sectors. The signal can easily be observed, and typically distinguished from a SM Higgs sector with only modest integrated luminosity, $\mathcal{O}(100) \mathrm{fb}^{-1}$, via study of the transverse mass distribution.

\section{MULTIPLE HIGGS DOUBLETS}

The continuum model [14] is merely a generalization of the SM to an arbitrary number of $S U(2)_{L}$ Higgs doublets. ${ }^{1}$ The vacuum expectation values $v_{i}=C_{i} v$, are bound by the measurement

$$
\sum_{i} v_{i}^{2}=v^{2} \sum_{i} C_{i}^{2} \geqslant v^{2} \equiv(246 \mathrm{GeV})^{2}
$$

with the equal sign $\Sigma C_{i}^{2}=1$ in the case of only singlet and doublet Higgs fields which we consider in this paper. Each doublet gives rise to one $C P$-even neutral Higgs boson $h_{i}$. There are, in addition, a large number of charged and $C P$ odd neutral Higgs bosons which can all be sufficiently heavy as to be unobservable. The question arises: given that none of the heavy states could be found, can LHC discover any of the $h_{i}$, and if so can it distinguish them from the SM Higgs boson?

The gauge couplings of the Higgs boson to weak bosons is proportional to $g v$, therefore it is modified for the $C P$-even Higgs boson of each doublet by the single factor $C_{i}$. The partial width of Higgs bosons to weak bosons is then $C_{i}^{2} \Gamma_{W, Z}^{\mathrm{SM}}$. A further constraint from electroweak precision data is [15]

$$
\sum_{i} C_{i}^{2} m_{h_{i}}^{2}=\left\langle M^{2}\right\rangle \lesssim(200 \mathrm{GeV})^{2}
$$

which also holds for any general supersymmetric Higgs sector [16].

Each SM fermion gets its mass in small pieces from the many Higgs fields: $m_{f}=\Sigma_{i} Y_{f}^{i} v_{i}$. In general, these Yukawa couplings need not be equal. In the minimal model, which assumes that ultimately the flavor sector comes from some universal condition, each $S U(2)_{L}$ Higgs doublet experiences the same set of Yukawa couplings $m_{f}=Y_{f} \Sigma_{i} v_{i}$. Matching the universal Yukawa coupling $Y_{f}$ with its SM counterpart we are left with $Y_{f}^{\mathrm{SM}}=Y_{f} \Sigma_{i} C_{i}$. Hence, the Higgs' partial widths to fermions acquire scaling factors $C_{i}^{2} /\left(\Sigma_{i} C_{i}\right)^{-2}$. The Higgs decay properties are particularly close to the SM properties in the limit of $N$ Higgs fields with identical vacuum expectation values $v_{i}=v / N$. For each partial width to $W, Z$ bosons becomes $\Gamma_{W, Z}=\Gamma_{W, Z}^{S M} / N^{2}$. The partial widths to fermions are scaled by the same factor $\Gamma_{f}=\Gamma_{f}^{\mathrm{SM}} / N^{2}$. Thus, to leading order all branching fractions for one of the many

\footnotetext{
${ }^{1}$ Additional singlets may also appear, but we disallow the presence of Higgs triplets which violate the custodial $S U(2)$ symmetry.
}

Higgs fields are identical to the SM values for the same Higgs mass; only the total width is suppressed by a factor $N^{-2}$.

The limiting case of a large number of Higgs fields is a Higgs continuum [16]. In this case, the distribution of vacuum expectation values becomes a continuous quantity, $C\left(m_{h}\right)$, expressed as a function of the physical Higgs boson mass. The sum rules in this case become

$$
\int d m_{h} C\left(m_{h}\right)^{2}=1, \int d m_{h} C\left(m_{h}\right)^{2} m_{h}^{2} \lesssim(200 \mathrm{GeV})^{2}
$$

As pointed out in Ref. [16], the weak production cross section for any point in the physical Higgs continuum is minimized when all the couplings to gauge bosons are set equal. This is precisely the continuum limit of the model with $N$ equal values $v_{i}$, which leaves the Higgs branching fractions unaffected to leading order. Taking the direct LEP constraints [17] together with the precision data, we find a scenario which is maximally likely to escape detection: $C\left(m_{h}\right)=C_{0}$ for $70 \mathrm{GeV} \leqslant m_{h} \leqslant 300 \mathrm{GeV}$ and $C\left(m_{h}\right)=0$ elsewhere. We adopt this case to illustrate our analysis, then slightly vary this assumption in Sec. III B.

\section{THE WEAK BOSON FUSION PROCESS}

We simulate $p p$ collisions at the LHC, $\sqrt{s}=14 \mathrm{TeV}$, for the final state $\ell_{1}^{+} \ell_{2}^{-} j j p_{T}$, calculating all signal and background cross sections with full tree level matrix elements for the contributing subprocesses. $\ell_{1}, \ell_{2}$ are any combination of $e$ and $\mu$, which are easily identified by the detectors with high efficiency. We employ CTEQ6L1 parton distribution functions [18] throughout. Unless otherwise noted the factorization scale is chosen as $\mu_{f}=\min \left(p_{T}\right)$ of the defined jets. The signal is calculated with matrix elements constructed by Madgraph [19], including exact matrix elements for the decay $H \rightarrow W^{+} W^{-} \rightarrow \ell_{1}^{+} \ell_{2}^{-} \nu_{1} \bar{\nu}_{2}$ to maintain the decay helicity correlations [2] on which the analysis rests.

The backgrounds consist of both QCD and EW processes leading to two far forward/backward jets, with a central pair of oppositely charged leptons $e$ or $\mu$, and large missing transverse energy. Although QCD processes with the same number of final state partons have much larger cross sections than the counterpart EW processes, in the region of phase space with two tagging jets the EW contribution can easily be of the same size as the QCD component. We thus consider the QCD processes $t \bar{t}+$ jets, $W^{+} W^{-} j j$ and $\tau^{+} \tau^{-} j j$, and the EW processes $W^{+} W^{-} j j$ and $\tau^{+} \tau^{-} j j[3]$, including correction factors for off-shell top quark effects $[4,20]$.

We also include here for the first time as a background $W$-fusion single-top production [21], $p p \rightarrow t b j$, where the light jet and hard-scattered $b$ jet appear as the far forward/ backward tagging jets, the top quark decays semileptonically, and its daughter $b$ quark also decays semileptonically. We use exact matrix elements for the $b$ decay to both charm and up quarks. 


\section{A. Basic kinematic cuts and jet selection}

The characteristics of WBF Higgs boson production are a pair of very far forward/backward tagging jets with significant transverse momentum and large invariant mass between them. Furthermore, the Higgs boson is produced centrally, and the decay products will therefore typically lie between the tagging jets, independent of the Higgs boson decay mode. Since the only modification to our signal here is a spectrum of Higgs boson masses rather than a single resonance, we employ the same optimized jet cuts as in the experimental simulation presented of Ref. [6]. These do not vary significantly from those of the original study [3], but do include more current understanding of detector and trigger requirements:

$$
\begin{gathered}
p_{T_{j}}>40,20 \mathrm{GeV}, \quad\left|\eta_{j}\right|<4.9, \Delta R_{j j}>0.4, \\
\eta_{j, \min }<\eta_{\ell}<\eta_{j, \max }, \quad \eta_{j_{1}} \cdot \eta_{j_{2}}<0 \\
\left|\eta_{j_{1}}-\eta_{j_{2}}\right|>3.8, \quad m_{j j}>550 \mathrm{GeV}
\end{gathered}
$$

The top pair background will frequently contain an extra central $b$ jet with $p_{T_{b}}>20 \mathrm{GeV}$ and $\left|\eta_{b}\right|<2.5$. We veto these in the same manner as in Ref. [6].

Although we are now examining a continuum of Higgs boson masses, they decay in the same manner as in the previous studies. The lepton cuts used therein take into account the detector observability and the angular correlations of the decay. Since neither change fundamentally, we also use the same lepton cuts as in Ref. [6]: ${ }^{2}$

$$
\begin{gathered}
p_{T_{\ell}}>15,20 \mathrm{GeV},\left|\eta_{\ell}\right| \leqslant 2.5, \triangle R_{j \ell}>0.4, \\
p_{T_{\ell}}<120 \mathrm{GeV}, \quad \cos \theta_{e \mu}>0.2, \quad \phi_{e \mu}<1.05, \\
\triangle R_{e \mu}<1.8, \quad m_{e \mu}<85 \mathrm{GeV} .
\end{gathered}
$$

Along with the $\triangle R_{j \ell}$ cut is the lepton isolation criteria for the semileptonic $b$ decay in $t \bar{t} j$ events: the hadronic remnant must have $p_{T}<3 \mathrm{GeV}$ if it lies within a cone of $\triangle R<0.4$ of the lepton.

The real tau backgrounds are large, especially the QCD component, but are reducible by reconstructing these taus $[22,5]$. In the collinear decay approximation, the fraction of tau energy that each charged lepton takes with it in the decay $\left(x_{\tau}\right)$ is solved for the actual charged lepton momenta and the missing energy in the transverse directions. Events with real tau pairs typically have $\vec{p}_{T}$ lying between the charged lepton flight directions; leptonic $W W$ events typically do not, and will most often give a fake-tau reconstruction with negative $x_{\tau}$ values. We therefore reject events with two positive $x_{\tau}$ values and an invariant tau pair mass of $m_{Z} \pm 25 \mathrm{GeV}[3,4]$.

\footnotetext{
${ }^{2}$ A separate parton level study [4] modified these cuts to optimize for a lighter Higgs boson, but in this analysis we focus on large Higgs boson masses, as will become obvious later. We are use the cuts of Ref. [4] for the same-flavor lepton channel.
}

QCD $b \bar{b} j j$ events with dual semileptonic $b$ decays constitutes a very large background to EW WWjj events [4]. However, they typically give a small transverse momentum for the reconstructed Higgs boson, because the leptons do not pass the isolation cut from the charm quark unless the parent $b$ quark is soft. We therefore employ dual twodimensional cuts [4] which suppress the $b \bar{b} j j$ background by more than two orders of magnitude:

$$
\triangle \phi_{\ell \ell, \mathrm{miss}}+1.5 p_{T_{H}}>180, \quad 12 \Delta \phi_{\ell \ell, \mathrm{miss}}+p_{T_{H}}>360 .
$$

We then reduce QCD $b \bar{b} j j$ events to an insignificant level by imposing a conditional cut on the transverse momentum [4]:

$$
\not p_{T}>20 \mathrm{GeV} \text { provided } p_{T_{H}}<50 \mathrm{GeV},
$$

where $p_{T_{H}}=\left|\vec{p}_{T}\left(\ell^{+}\right)+\vec{p}_{T}\left(\ell^{\prime}\right)+\vec{p}_{T}\right|$. Both cuts result in only trivial signal rejection.

In the case of same lepton flavors, low mass $\ell^{+} \ell^{-}$pairs originating from $\gamma^{\star} \rightarrow \ell^{+} \ell^{-}$exhibit a large cross section and are suppressed by requiring that the dilepton invariant mass is larger than $10 \mathrm{GeV}$ [4]. To reduce the background arising from $\ell^{+} \ell^{-} j j$, where the missing transverse momentum is generated by detector effects, we further require the missing transverse momentum to be larger than $30 \mathrm{GeV}$ [4]. In brief, we impose the additional cuts on the same flavor final state:

$$
m_{\ell \ell}>10 \mathrm{GeV} \text { and } \not b_{T}>30 \mathrm{GeV} .
$$

Finally, we note that the single-top background is dominated by the semileptonic $b$ decay $b \rightarrow u \ell \nu$, rather than $c \ell \nu$, although the CKM matrix element is larger in the latter case. Because the kinematics of the lighter quark decay allows more momentum of the light quark transverse to the $b$ flight direction, it can more often lie outside the lepton isolation cone.

\section{B. Discovery potential of the LHC}

The size of the signal which could be observed at the LHC in the channel described above depends critically on the continuum mass window. We know that for flat $C\left(m_{h}\right)$ $=C_{0}$ the branching fraction to $W W$ is reduced for small Higgs boson masses. In other words, the continuum signal at low masses will be suppressed by the $W W$ branching fraction, despite the larger Higgs boson production cross section. Large continuum masses will be production phase space suppressed, the $Z Z$ branching fraction becomes significant, and the selection cuts are not optimized for $m_{h} \gtrsim 200 \mathrm{GeV}$. Hence, even a model with a wide range of continuum masses will still leave the event sample dominated by Higgs boson masses around the $W W$ threshold. In Fig. 1 we show the contribution from a continuum Higgs sector, $70 \leqslant m_{h}$ $\leqslant 300 \mathrm{GeV}$, to the total $W^{+} W^{-} j j$ cross section. As expected, the bulk of the signal events originate from Higgs boson masses around $2 M_{W}$.

As the final state events contain two unobservable neutrinos and are thus not fully reconstructible, we cannot directly 


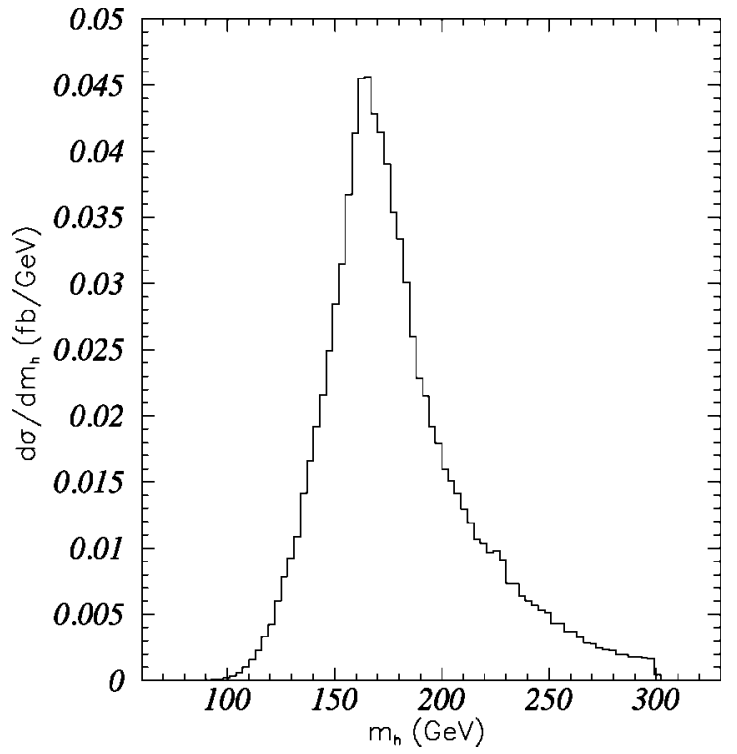

FIG. 1. Distribution of the signal cross section after all cuts, as a function of the continuum Higgs boson mass, for the case 70 $\leqslant m_{h} \leqslant 300 \mathrm{GeV}$, as discussed in the text.

observe the $m_{h}$ distribution in Fig. 1. However, the general behavior can be read off the measurable $W W$ transverse mass distribution [3],

$$
\begin{aligned}
m_{T}^{2} \equiv m_{T, W W}^{2}= & \left(\sqrt{\vec{p}_{\ell \ell, T}^{2}+m_{\ell \ell}^{2}}+\sqrt{\vec{p}_{\nu \nu, T}^{2}+m_{\nu \nu}^{2}}\right)^{2} \\
& -\left(\vec{p}_{\ell \ell, T}+\vec{p}_{\nu \nu, T}\right)^{2},
\end{aligned}
$$

which for a single resonance exhibits a sharp edge near the invariant $W W$ mass $m_{W W}$. The neutrino pair transverse momentum is determined as the missing transverse momentum $\vec{p}_{\nu \nu, T}=\vec{p}_{T}$. The invariant mass of the neutrino pair cannot be observed, so we replace it with the lepton pair invariant mass $m_{\nu \nu} \sim m_{\ell \ell}$ [3], an approximation which is exact for $m_{h}$ $=2 M_{W}$, and very good over the intermediate Higgs boson mass range. ${ }^{3}$ On average the two kinds of $W$ decay fermions will give the same distributions, even though on an event-byevents basis this approximation violates the condition $m_{T}$ $\leqslant m_{W W}$. Detector effects, mostly mismeasurement of the missing transverse momentum due to the presence of the hadronic forward tagging jets, will dull the edge in $m_{T}$ somewhat, but the distribution remains extremely important $[6,7]$.

For a continuum model we indeed observe a transverse mass peak around the $W W$ threshold, but with a considerable tail extending to larger $m_{T}$ values and therefore larger continuum Higgs boson masses. For a flat continuum mass spectrum, the position of the peak in Fig. 2 is approximately the peak position of the $m_{T}$ curve given by a SM Higgs whose mass maximizes the function $\sigma_{H j j}^{S M}\left(m_{h}\right) \times B_{W W}\left(m_{h}\right)$.

${ }^{3}$ In the limit $m_{\ell \ell}, m_{\nu \nu} \rightarrow 0$ the $W W$ transverse mass can be written as $m_{T, W W}^{2}=2 p_{\ell \ell, T} \not p_{T}(1-\cos \Delta \Phi)$. This alternative definition can be used to distinguish signal and background, but does not work particularly well in distinguishing continuum Higgs sectors from the SM.

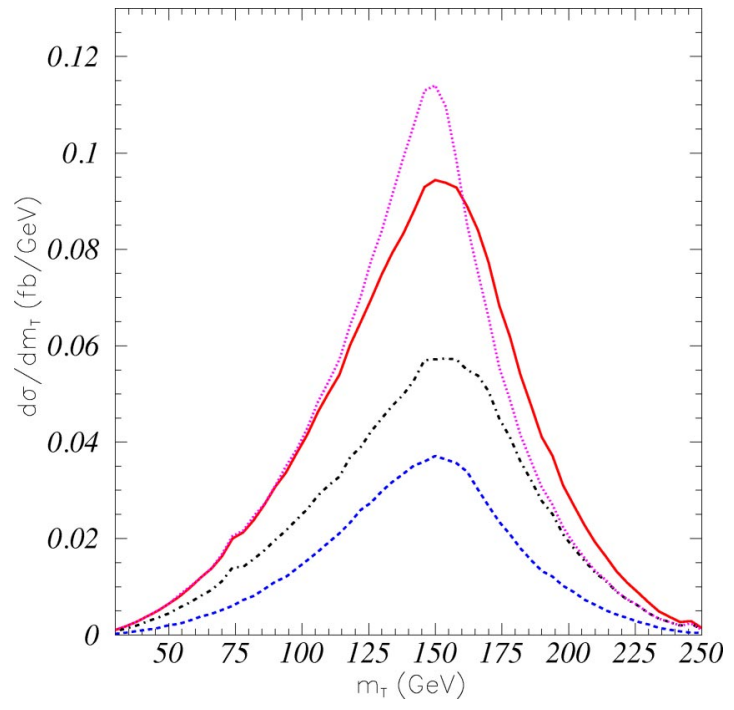

FIG. 2. Transverse mass $m_{T}$ spectrum for the continuum model signal (dashed), background (dash-dotted), and continuum signal plus background (solid). For comparison we also show the $m_{h}$ $=155 \mathrm{GeV}$ SM signal plus background case, normalized to have the same signal cross section as the continuum model.

We can see from Table I that all backgrounds are very much under control, even with a reduced signal rate compared to the SM Higgs. In the intermediate mass range a SM Higgs could be observed with $S / B=2-6$. Now, with a wide mass window, this fraction is reduced by roughly a factor 3 . Because the luminosity required to find a SM Higgs boson of mass $160 \mathrm{GeV}$ at $5 \sigma$ in this channel is less that $5 \mathrm{fb}^{-1}$, the luminosity required to discover a continuum Higgs sector is still small. For a flat distribution $\left[C\left(m_{h}\right)=C_{0}\right]$ over the range $70 \leqslant m_{h} \leqslant 300 \mathrm{GeV}, 14 \mathrm{fb}^{-1}$ are necessary to observe a $5 \sigma$ signal with a transverse mass peak as shown in Fig. 2 .

\section{Distinguishing continuum from single resonance}

While continuum Higgs sector discovery potential depends strongly on the production rate and Higgs branching fraction to $W$ bosons, $B_{W W}\left(m_{h}\right)$, these numbers depend strongly on the precise shape of $C\left(m_{h}\right)$. We propose to use the $W W$ transverse mass distribution shape to distinguish SM from continuum Higgs sectors. For reasons discussed in Sec. III B, a continuum Higgs sector exhibits a nearly symmetric distribution about the peak, with the maximum around $150 \mathrm{GeV}$. For small masses the rate is cut off by a diminishing branching fraction, and for high masses by the increasingly restrictive production phase space (cf. Fig. 1). In contrast, a SM Higgs $m_{T}$ distribution is much more Jacobian. After determining the $m_{T}$ maximum we define the asymmetry

$$
\mathcal{A}=\frac{\sigma\left(m_{T}<m_{T}^{\max }\right)-\sigma\left(m_{T}>m_{T}^{\max }\right)}{\sigma\left(m_{T}<m_{T}^{\max }\right)+\sigma\left(m_{T}>m_{T}^{\max }\right)} \equiv \frac{L-H}{L+H}=\frac{L-H}{N},
$$

where $L(H)$ is the number of signal events with $m_{T}<$ $(>) m_{T}^{\max }$, and $N$ is the total number of signal events. We 
TABLE I. Signal and background cross sections ( $\mathrm{fb}$ ) after all kinematic cuts as discussed in the text. The first two columns refer to different-flavor $(e \mu)$ final states, while the last two columns give results for the same-flavor $(l l=e e, \mu \mu)$ sample. The first row is the continuum Higgs model considered, and we show the SM signal which has the same peak value of $m_{T}(W W)$ in the second row for comparison. The last three rows display the total background for each channel, with and without the minijet veto; the signal-to-background ratio for the continuum model; and the required integrated luminosity to observe the continuum model signal over the SM background.

\begin{tabular}{llccc}
\hline \hline Channel & $e^{ \pm} \mu^{\mp}$ & $e^{ \pm} \mu^{\mp}$ w/minijet veto & $e^{ \pm} e^{\mp}, \mu^{ \pm} \mu^{\mp}$ & $e^{ \pm} e^{\mp}, \mu^{ \pm} \mu^{\mp}$ w/minijet veto \\
\hline $70<m_{h}<300 \mathrm{GeV}$ & 1.90 & 1.69 & 1.56 & 1.39 \\
$\mathrm{SM}, m_{h}=155 \mathrm{GeV}$ & 5.60 & 4.98 & 4.45 & 3.96 \\
\hline $\bar{t}$ & 0.086 & 0.025 & 0.086 & 0.025 \\
$t \bar{t} j$ & 7.59 & 2.20 & 6.45 & 1.87 \\
$t \bar{t} j j$ & 0.83 & 0.24 & 0.72 & 0.21 \\
single top $(t b j)$ & 0.020 & 0.015 & 0.016 & 0.012 \\
$b \bar{b} j j$ & 0.010 & 0.003 & 0.003 & 0.001 \\
QCD $W W j j$ & 0.448 & 0.130 & 0.390 & 0.113 \\
EW $W W j j$ & 0.269 & 0.202 & 0.239 & 0.179 \\
QCD $\tau \tau j j$ & 0.128 & 0.037 & 0.114 & 0.033 \\
EW $\tau \tau j j$ & 0.017 & 0.013 & 0.016 & 0.012 \\
QCD $\ell \ell j j$ & - & - & 0.114 & 0.033 \\
EW $\ell \ell j j$ & - & - & 0.011 & 0.008 \\
\hline Total bkg & 9.40 & 2.87 & 8.04 & 2.49 \\
$S / B$ & $1 / 5.0$ & $1 / 1.7$ & $1 / 5.1$ & $1 / 1.8$ \\
$\mathcal{L}_{5 \sigma}^{\text {obs }}\left(\mathrm{fb}{ }^{-1}\right)$ & 65 & 25 & 82 & 32 \\
\hline \hline
\end{tabular}

expect the asymmetry to be small if the observed Higgs signal comes from a continuum or multiple-fields model.

To quantify the impact of this asymmetry we again assume a flat distribution, $C\left(m_{h}\right)=C_{0}$, over $70 \leqslant m_{h}$ $\leqslant 300 \mathrm{GeV}$. Assuming that the total rate cannot serve as a means to distinguish the continuum model from the SM Higgs sector, we normalize the $155 \mathrm{GeV}$ Higgs SM rate to the continuum rate, after all cuts. This could, e.g., be realized by an overall suppression of the $H W W$ coupling, such as occurs via $\sin (\beta-\alpha)$ in a two Higgs doublet model. We then evaluate the transverse $W W$ mass asymmetry $\mathcal{A}$ for signal and background and compute the luminosity required to observe a continuum signal five standard deviations away from the $\mathrm{SM}$ value.

For uncorrelated statistical errors $\sigma_{l}\left(\sigma_{h}\right)$ on $L(H)$, the statistical significance of $\mathcal{A}$ is [23]

$$
\sigma_{\mathcal{A}}=\frac{2 L H}{N^{2}} \sqrt{\left(\frac{\sigma_{L}}{L}\right)^{2}+\left(\frac{\sigma_{H}}{H}\right)^{2}}=\frac{1-\mathcal{A}^{2}}{2} \sqrt{\frac{1}{L}+\frac{1}{H}},
$$

where the last part of the relation holds if the number of events satisfies Poisson statistics.

We show our results for several choices of continuum Higgs model parametrizations in Table II. Any continuum sector would be very easy to observe, typically requiring only around $10 \mathrm{fb}^{-1}$ to observe, although a case designed to be difficult because of low branching ratio to $W W, 70<m_{h}$ $<150 \mathrm{GeV}$, might require up to $50 \mathrm{fb}^{-1}$ to observe at $5 \sigma$.
Discriminating a continuum sector from a SM Higgs presenting a peak in the same position in $m_{t}$ is viable, but requires significant additional integrated luminosity, typically $\mathcal{O}(100) \mathrm{fb}^{-1}$. The difficult cases, requiring close to ten times that amount of data, are where the continuum is spread over very low masses, typically $m_{h}<150 \mathrm{GeV}$, such that very little of the total cross section decays to $W W$ final states and the events lie below the nearly symmetric peak in $\sigma_{H j j}^{S M}\left(m_{h}\right) \times B_{W W}\left(m_{h}\right)$.

Note that we could have fitted the continuum $m_{T}$ line shape to a SM Higgs boson mass, but we prefer to determine the SM mass to compare with from the peak of the transverse mass distribution. The only problem we can think of is the mass distribution $C\left(m_{h}\right)$ mimicking the transverse mass distribution of the SM Higgs boson. However, this only impacts the distinction from the SM and leaves the discovery prospects unaffected.

\section{REMAINING LHC HIGGS BOSON SEARCHES}

Having described how the multi Higgs resonance model is naturally picked up by the WBF Higgs search with decay to $W$ bosons, we briefly describe how the other LHC Higgs boson search channels would be affected by this model.

Weak boson fusion. The decay to $W$ pairs is not the only decay channel proven capable of giving rapid Higgs boson discovery in WBF processes. The decay to tau leptons in this production channel is in fact the most solid channel in the MSSM, where it can even distinguish between the two $\mathrm{CP}$ even scalar mass peaks $[10,5]$. However, a mass peak is pre- 
TABLE II. The asymmetry defined in Eq. (10) for SM Higgs signals and various choices of the continuum Higgs sector. We choose the SM Higgs mass of each pairing to give the same peak position in $m_{T}$ as the corresponding continuum model. The first pair corresponds to the widest mass range; all choices assume $C\left(m_{h}\right)=C_{0}$. The cross sections include all cuts and minijet veto survival probabilities. $\mathcal{L}_{5 \sigma}^{\text {obs }}$ is the luminosity required to observe the signal above background ("detectability"). $\mathcal{L}_{5 \sigma}^{\mathcal{A}}$ is the luminosity required to distinguish the signal, using the $m_{T}$ asymmetry $\mathcal{A}$, as coming from a continuum model or a $150-170 \mathrm{GeV} \mathrm{SM}$ Higgs boson, depending on the case ("distinguishability").

\begin{tabular}{|c|c|c|c|c|}
\hline Higgs boson mass (window) & $\sigma(\mathrm{fb})$ & $\mathcal{L}_{5 \sigma}^{\mathrm{obs}}\left(\mathrm{fb}^{-1}\right)$ & $\mathcal{A}$ & $\mathcal{L}_{5 \sigma}^{\mathcal{A}}\left(\mathrm{fb}^{-1}\right)$ \\
\hline SM: $155 \mathrm{GeV}$ & 14.3 & $<5$ & 0.186 & \multirow{2}{*}{238} \\
\hline $70-300 \mathrm{GeV}$ & 8.40 & 14 & 0.076 & \\
\hline SM: $155 \mathrm{GeV}$ & 14.3 & $<5$ & 0.210 & \multirow{2}{*}{161} \\
\hline $100-270 \mathrm{GeV}$ & 9.40 & 8 & 0.085 & \\
\hline SM: $155 \mathrm{GeV}$ & 14.3 & $<5$ & 0.241 & \multirow{2}{*}{84} \\
\hline $130-240 \mathrm{GeV}$ & 11.0 & 4 & 0.081 & \\
\hline SM: $160 \mathrm{GeV}$ & 16.7 & $<5$ & 0.231 & \multirow[t]{2}{*}{145} \\
\hline $185-300 \mathrm{GeV}^{\mathrm{a}}$ & 7.37 & 32 & 0.082 & \\
\hline SM: $170 \mathrm{GeV}$ & 16.2 & $<5$ & 0.254 & \multirow[t]{2}{*}{139} \\
\hline $185-210 \mathrm{GeV}$ & 10.1 & 6 & 0.125 & \\
\hline SM: $155 \mathrm{GeV}$ & 14.3 & $<5$ & 0.211 & \multirow{2}{*}{810} \\
\hline $70-180 \mathrm{GeV}$ & 9.43 & 8 & 0.155 & \\
\hline SM: $150 \mathrm{GeV}$ & 12.6 & $<5$ & 0.078 & \multirow{2}{*}{897} \\
\hline $70-150 \mathrm{GeV}$ & 6.92 & 52 & 0.141 & \\
\hline
\end{tabular}

${ }^{\mathrm{a}}$ Satisfies the slightly weaker constraint $\left\langle M^{2}\right\rangle \lesssim(214 \mathrm{GeV})^{2}$ [see Eq. (2)].

cisely where the multi-resonance model avoids discovery. No matter how wide the spread in Higgs boson masses becomes, the WBF tau decay channel can see only the range between about 115 and $145 \mathrm{GeV}$. In that range the signal would be approximately flat in tau pair invariant mass, appearing to be an unexplained enhancement in the $Z \rightarrow \tau \tau$ Breit-Wigner tail above the peak. WBF production and decay to photon pairs is also a useful channel [24], but similarly would give an essentially flat invariant mass distribution. Because this channel yields very few events, it works in the SM case only because the detectors have a very narrow resolution in photon pair invariant mass. Spreading a very small number of signal events out from a $2 \mathrm{GeV}$ window to over a $\sim 40 \mathrm{GeV}$ range would result in complete loss of detectability. Seeing a Higgs signal in the WBF channel decaying to a $W$ pair and not seeing it in the $\tau$ decays in an accessible mass range would point to a Higgs sector beyond the SM, possibly a continuum model.

Gluon fusion. The two most powerful decay channels for measuring the Higgs boson mass are photon pairs and the "golden mode" of four leptons (via a $Z$ boson pair) $[8,9,25]$, produced in gluon fusion [26]. Unfortunately, this feature turns into the worst disadvantage once the mass window in the analysis has to be several tens of GeV. A side-band analysis of the continuum background will not work because there is no true resonance, and the significance of the signal will vanish. On the other hand, we expect the $W W$ decay would work similarly well as in the WBF process. The $\ell \ell \nu \nu$ invariant mass shown in Fig. 3 of Ref. [2] will change in analogy to the transverse mass in the WBF channel, but without a detailed simulation (beyond the scope of this work) it is not clear if the shape change would allow SM vs multiresonance Higgs sector discrimination.

Top quark associated production. Despite the relatively small cross section [27], top quark events are highly distinctive and associated production with a Higgs boson has received much interest as a result. For $m_{h}<135 \mathrm{GeV}$ the dominant decay mode is to a pair of bottom quarks, which appears as a very small peak near the $t \bar{t} b \bar{b}$ continuum peak [28]. Such a signal spread out via continuum Higgs production would obviously be immediately lost in the background, as would decay to photon pairs, as in WBF or inclusive Higgs boson production. For $m_{h}>135 \mathrm{GeV}$ the dominant decay is to $W$ pairs. This will be a very useful channel for measuring the top Yukawa coupling [29], if the Higgs sector is SM-like and $m_{h}>135 \mathrm{GeV}$. However, the multi-lepton final states used in this planned analysis do not permit full reconstruction, so the Higgs boson mass is never identified. Because of the lack of need for such a resonance, this channel should work nearly as well as in the SM case, although a detailed investigation is again beyond the scope of our present work. 
Weak boson associated production. If the Higgs boson is produced in association with a weak gauge boson it can be searched for in the photon decay channel $[8,9]$ because of the very narrow resonance peak distinguishable above a large background. However, just as in the case of decay to photon pairs in all the other production channels, there is no longer any resonance, and the signal will be lost in the continuum background. There is also a small signal for $H \rightarrow b \bar{b}$ in the SM [30], but as with $t \bar{t} H$ production the peak is similarly lost in the very large $W b \bar{b}$ QCD continuum [31]. Thus, none of these modes would be observable.

\section{CONCLUSIONS}

We have shown that continuum Higgs models do not present any problem for LHC Higgs discovery. If the coupling to gauge bosons is spread over a mass range $m_{h} \sim 100-300 \mathrm{GeV}$ these continuum Higgs boson events will automatically appear in the searches for Higgs bosons produced in weak boson fusion, with subsequent decay to $\ell^{+} \ell^{-}$and missing transverse energy via $W$ pairs. The broad nature of the Higgs resonance has no major impact on this search channel, because the corresponding standard model search channel already benefits from hugely suppressed backgrounds, typically much less than the level of the signal. In the case of a continuum Higgs sector, the signal to background ratio typically is in the range $1 / 1-1 / 3$, still extremely good. Discovery of the most difficult case studied would require only about $50 \mathrm{fb}^{-1}$, and almost all cases require less than $30 \mathrm{fb}^{-1}$. Our analysis is not subject to detector uncertainties, such as poorly modeled $\boldsymbol{E}_{T}$ resolution, or identification and measurement of the tagging jets, which are known with less certainty for high-luminosity running; only lowluminosity running is needed for discovery in this channel.

Using the nearly symmetric behavior of the transverse $W W$ mass for continuum Higgs sectors, we can distinguish these models from the SM without relying on the total rate. These features are largely independent of the detailed spectrum $C\left(m_{h}\right)$ of the continuum Higgs sector and of the distribution of the coupling $g_{W W h}\left(m_{h}\right)$. The integrated luminosity required to distinguish such a sector from the SM is typically $\mathcal{O}(100) \mathrm{fb}^{-1}$, but can be as large as $900 \mathrm{fb}^{-1}$ for the most difficult case studied, where the continuum is spread over a mass region less than $150 \mathrm{GeV}$.

Search strategies not using $W W$ final states will almost universally be unable to see these states, as they require a sharp peak in a mass spectrum, which does not exist in the flat-spectrum continuum models. Such an observation could be interpreted as a sign of a Higgs sector more complex than that of the SM, e.g. a continuum sector.

\section{ACKNOWLEDGMENTS}

This research was supported in part by Fundação de Amparo à Pesquisa do Estado de São Paulo (FAPESP), by Conselho Nacional de Desenvolvimento Científico e Tecnológico $(\mathrm{CNPq})$, and by Programa de Apoio a Núcleos de Excelência (PRONEX).
[1] LEPEWWG, D. Abbaneo et al., hep-ex/0212036.

[2] M. Dittmar and H.K. Dreiner, Phys. Rev. D 55, 167 (1997).

[3] D. Rainwater and D. Zeppenfeld, Phys. Rev. D 60, 113004 (1999); 61, 099901(E) (2000).

[4] N. Kauer, T. Plehn, D. Rainwater, and D. Zeppenfeld, Phys. Lett. B 503, 113 (2001).

[5] D. Rainwater, D. Zeppenfeld, and K. Hagiwara, Phys. Rev. D 59, 014037 (1999); T. Plehn, D. Rainwater, and D. Zeppenfeld, ibid. 61, 093005 (2000).

[6] C. Buttar, R. Harper, and K. Jakobs, ATL-PHYS-2002-033.

[7] S. Asai et al., ATL-PHYS-2003-005; B. Mellado, ATL-CONF2002-004; N. Akchurin et al., CMS-NOTE-2002/066; G. Azuelos and R. Mazini, ATL-PHYS-2003-004; K. Cranmer et al., ATL-PHYS-2003-008 and ATL-PHYS-2003-007.

[8] ATLAS TDR, report CERN/LHCC/99-15 (1999).

[9] CMS TP, report CERN/LHCC/94-38 (1994).

[10] T. Plehn, D. Rainwater, and D. Zeppenfeld, Phys. Lett. B 454, 297 (1999).

[11] U. Ellwanger, J.F. Gunion, C. Hugonie, and S. Moretti, hep-ph/0305109.

[12] T. Binoth and J.J. van der Bij, hep-ph/9409332; Z. Phys. C 75, 17 (1997).

[13] O.J.P. Éboli and D. Zeppenfeld, Phys. Lett. B 495, 147 (2000).

[14] J.R. Espinosa and J.F. Gunion, Phys. Rev. Lett. 82, 1084 (1999)

[15] J.F. Gunion, hep-ph/0106154.
[16] J.R. Espinosa and M. Quiros, Phys. Rev. Lett. 81, 516 (1998).

[17] LEP Higgs Working for Higgs boson searches Collaboration, R. Barate et al., Phys. Lett. B 565, 61 (2003).

[18] J. Pumplin et al., J. High Energy Phys. 07, 012 (2002).

[19] T. Stelzer and W.F. Long, Comput. Phys. Commun. 81, 357 (1994).

[20] N. Kauer, Phys. Rev. D 67, 054013 (2003); N. Kauer and D. Zeppenfeld, ibid. 65, 014021 (2002).

[21] S.S.D. Willenbrock and D.A. Dicus, Phys. Rev. D 34, 155 (1986).

[22] R.K. Ellis, I. Hinchliffe, M. Soldate, and J.J. van der Bij, Nucl. Phys. B297, 221 (1988).

[23] L. Lyons, Statistics for Nuclear and Particle Physicists (Cambridge University Press, Cambridge, England, 1986).

[24] D. Rainwater and D. Zeppenfeld, J. High Energy Phys. 12, 005 (1997).

[25] K. Cranmer, B. Mellado, W. Quayle, and S. L. Wu, ATLPHYS-2003-025.

[26] M. Spira, A. Djouadi, D. Graudenz, and P.M. Zerwas, Nucl. Phys. B453, 17 (1995); R.V. Harlander and W.B. Kilgore, Phys. Rev. Lett. 88, 201801 (2002); C. Anastasiou and K. Melnikov, Nucl. Phys. B646, 220 (2002); V. Ravindran, J. Smith, and W.L. van Neerven, ibid. B665, 325 (2003); Mod. Phys. Lett. A A 18, 1721 (2003).

[27] W. Beenakker et al., Phys. Rev. Lett. 87, 201805 (2001); S. Dawson, L.H. Orr, L. Reina, and D. Wackeroth, Phys. Rev. D 67, 071503 (2003). 
[28] V. Drollinger, T. Müller, and D. Denegri, hep-ph/0111312; J. Cammin and M. Schumacher, ATL-PHYS-2003-024.

[29] F. Maltoni, D. Rainwater, and S. Willenbrock, Phys. Rev. D 66, 034022 (2002); V. Kostioukhine, J. Leveque, A. Rozanov, and J. B. de Vivie, ATL-PHYS-2002-019.
[30] V. Drollinger, T. Müller, and D. Denegri, hep-ph/0201249; B.P. Kersevan and E. Richter-Was, Eur. Phys. J. C 25, 379 (2002).

[31] J. Campbell, R.K. Ellis, and D. Rainwater, Phys. Rev. D 68, 094021 (2003) 